Trauma Berufskrankh 2008 10 [Suppl 2]:200-202

DOI 10.1007/s10039-007-1315-3

Online publiziert: 26. April 2008

(c) Springer Medizin Verlag 2008

J. Engelhard

LKA 328 - Medicus, Landeskriminalamt, Berlin

\title{
Wann ist die ärztliche Abrechnung strafbar
}

Gerne werde ich bei derartigen Veranstaltungen gefragt, warum ich nicht gegen betrügerische Autohändler ermittle oder gegenüber Kfz-Werkstätten, die ihre Leistungen betrügerisch überhöht abrechnen. Sicherlich kann man sich diesem Themenbereich widmen, es befremdet mich aber ein wenig, gerade gegenüber einem Ärzteauditorium über derartige Betrügereien zu referieren. Natürlich kenne ich nicht die Gepflogenheiten von Ärzten bezüglich Fahrzeugerwerb oder instandsetzungen. Ich bin mir aber recht sicher, dass die Häufigkeiten diesbezüglicher Betrügereien und die damit verbundene potenzielle Schädigung um ein Vielfaches geringer sind als die Schädigung durch betrügerisch falsch abrechnende Fachkollegen, denn der betrügerisch abrechnende Arzt greift Quartal für Quartal in die Töpfe der Fachgruppen und entzieht dadurch Honorar, welches den ehrlich abrechnenden Fachkollegen zugestanden hätte. Es werden - zumindest in der vertragsärztlichen Versorgung - ausschließlich die Kollegen geschädigt.

\section{Gesetzliche Grundlagen und Beispiele}

Für den Abrechnungsbetrug sind letztendlich 2 Paragrafen des Strafgesetzbuchs einschlägig.

Zunächst ist der $\$ 263$ StGB zu nennen. Dieser stellt betrügerische Handlungen unter Strafe. Darunter ist zu verstehen, dass durch den Täter falsche Tatsachen erklärt bzw. richtige Tatsachen unterdrückt werden. Durch diese Verzerrung der Realität entsteht ein Irrtum, welcher dafür ausschlaggebend ist, dass gegenüber dem Täter Vermögen verfügt wird. Diese Verfügung wäre nicht erfolgt, wenn das Op- fer über die tatsächlichen Umstände informiert gewesen wäre. Beim Opfer tritt ein Schaden ein. Wichtig ist, dass der Geschehensablauf vom Täter beabsichtigt ist (subjektiver Tatbestand). In der Welt der medizinischen Leistungserbringung übersetzt bedeutet dies, dass der Leistungserbringer gegenüber einem Kostenträger die Vergütung einer Leistung deklariert. Dies erfolgt, indem er eine Gebührenordnungsposition nennt. In dieser ist festgelegt, welche Leistung erbracht wurde und wie diese zu vergüten ist, sei es nun in Eurobeträgen oder über eine relative Gewichtung in einem Punktesystem. Hat der Leistungserbringer gewisse Pflichten der Leistungsbeschreibung nicht erbracht, stünde ihm kein Leistungshonorar zu. Erfolgen trotzdem im Wissen der Nichtleistung die Anforderung der entsprechenden Gebührenordnungsposition und letztendlich auch deren Vergütung, wäre der Betrug vollendet.

Neben dem Betrug erlangt der \$266 StGB aufgrund der Rechtsprechung des Bundesgerichtshofs an Bedeutung und soll deshalb hier kurz thematisiert werden. Es handelt sich um den Tatbestand der Untreue. Sie sieht vor, dass dem Täter die Befugnis eingeräumt wurde, über fremdes Vermögen zu verfügen bzw. der Täter eine Vermögensbetreuungspflicht für fremdes Vermögen hat. Diese Befugnis wird vom Täter missbraucht bzw. es erfolgt durch die Verletzung der Vermögensbetreuungspflicht eine Schädigung des Vermögens. In die Diskussion kam der Untreuetatbestand durch eine Entscheidung des BGH (4 StR 239/03). Ein Arzt hatte GKV-Gefälligkeitsverschreibungen durchgeführt, die medizinisch nicht indiziert waren. Der Versicherte und auch der Arzt wurden zunächst vom zuständigen Landgericht wegen Betrugs verurteilt. 
Dieses Urteil wurde durch den BGH aufgehoben. Dieser sah vielmehr, dass sich der Arzt einer Untreue schuldig gemacht hatte. Der BGH führte hierzu aus:

„Nach den Prinzipien des kassenärztlichen Abrechnungssystems handelt der Vertragsarzt bei Ausstellung einer Verordnung - wie ausgeführt - als Vertreter der Krankenkasse, indem er an ihrer Stelle das Rahmenrecht des einzelnen Versicherten auf medizinische Versorgung konkretisiert. Der Kassenarzt darf allerdings den materiellen (und formellen) Rahmen der kassenärztlichen Versorgung nicht verlassen (vgl. nur BSGE 73, 271, 278, 281f.). Er darf deshalb Leistungen, die jenseits der Bandbreite offener Wertungen nach den Regeln der ärztlichen Kunst (vgl. Schwerdtfeger aaO, S. 101) eindeutig nicht notwendig, nicht ausreichend oder unzweckmäßig sind, nicht verordnen ( $\$ \$ 12$ Abs. 1 Satz 2, 70 Abs. 1 Satz 2 SGB V). Verschreibt der Kassenarzt dennoch ein Medikament zu Lasten der Krankenkasse, obwohl er weiß, dass er die Leistung - wie hier - im Sinne des $\$ 12$ Abs. 1 SGB V nicht bewirken darf, missbraucht er diese ihm vom Gesetz eingeräumten Befugnisse. Damit verletzt er seine Betreuungspflicht gegenüber dem betroffenen Vermögen der Krankenkasse."

Der Betrugsverdacht liegt erfahrungsgemäß immer dann vor, wenn durch einen Arzt eine Leistung abgerechnet wird, welche nicht gemäß der einschlägigen Leistungslegende vollständig erbracht wurde. Hier ist darauf abzustellen, dass der Kostenträger beim Abrechnen einer Gebührenordnungsposition von deren vollständiger Erbringung ausgeht. Es wäre zu keiner Leistungsvergütung gekommen, wenn die tatsächliche Mindererfüllung bekannt gewesen wäre.

Auch in der Person des Leistungserbringers kann eine Leistungsstörung liegen, welche zur Nichtabrechnungsfähigkeit einer Leistung führt und im Fall der Abrechnung den Betrugsverdacht begründet. Hier wäre als Beispiel die Tätigkeit eines Nicht-BG-Arztes anzuführen, welche durch den BG-Arzt als Praxispartner oder Partner in einem medizinischen Versorgungszentrum abgerechnet wurde. Auch hier geht der Kostenträger zunächst davon aus, dass der abrechnende Arzt die Leistung persönlich erbracht hat.

Trauma Berufskrankh 2008 · 10[Suppl 2]:200-202 DOI 10.1007/s10039-007-1315-3

(c) Springer Medizin Verlag 2008

\section{J. Engelhard \\ Wann ist die ärztliche Abrechnung strafbar}

\section{Zusammenfassung}

Im vorliegenden Beitrag werden die Erfahrungen des Landeskriminalamts Berlin bei Ermittlungsverfahren wegen Abrechnungsbetruges geschildert. Seit inzwischen 10 Jahren wird dieser Deliktbereich im LKA Berlin spezialisiert bearbeitet. Letztendlich sind für inn 2 Paragrafen des Strafgesetzbuchs einschlägig, §263 und §266 StGB. Generell kann man die Abrechnungsbetrügereien unter den ersten 3 in der Literatur aufgetretenen Deliktbeschreibungen ("goldene Drei") zusammenfassen: Luftabrechnung, Leistungssplitting und Leistungsaufwertung. Daneben gibt es noch

\section{Accounting fraud}

\section{Abstract}

The present paper describes experiences of investigations conducted by the Land Office of Criminal Investigation in Berlin (LKA) into accounting fraud. For the past 10 years, this type of criminal offence has been handled by specialist teams. Two paragraphs of the Penal Code, $\$ 263$ and $\$ 266$, apply directly to such offences. In general, accounting frauds can be subsumed under the first three descriptions of offences to occur in the literature (the "golden three"): invoicing of services never provided; separate invoices from more than one doctor involved in provision of the same service; and invoicing for more expen- vielfältige weitere Möglichkeiten, recht häufig sind auch Doppelabrechnungen. Im Rahmen von Ermittlungsverfahren kommt der ärztlichen Dokumentation entscheidende Bedeutung zu: Je umfangreicher dokumentiert wird, desto einfacher lassen sich Leistungserbringungen nachweisen.

\section{Schlüsselwörter}

Landeskriminalamt · Abrechnungsbetrug . Luftabrechnung · Leistungssplitting .

Leistungsaufwertung sive services than actually provided. In addition there are myriad other possibilities: double invoicing is quite common. In the context of the investigations the medical documentation becomes decisively important: the more comprehensive the records the simpler it is to prove what services have been provided.

\section{Keywords}

Land Office of Criminal Investigation . Accounting fraud $\cdot$ Invoicing of services never provided. Separate invoices of the same service $\cdot$ Invoicing for more expensive services 
Nicht nur in der ärztlichen Tätigkeit, sondern auch in anderen Leistungsbereichen kann sich ein Betrugsverdacht begründen, so z. B. bei der Abrechnung von Sachkosten. Diese dürfen als durchlaufende Kosten nur in der Höhe deklariert werden, in der sie tatsächlich anfallen. In der praktischen Arbeit fallen hier wiederholt Fälle auf, in denen Sachkosten überhöht abgerechnet werden, ein Beispiel ist der Zahnersatzskandal „Globudent", in dem Laborleistungen bundesweit überteuert durch das zahntechnische Institut abgerechnet wurden. Diese Rechnungen wurden von betroffenen Zahnärzten an die Kostenträger weitergereicht. Später erhielten diese Zahnärzte Kick-Back-Zahlungen ausgehändigt.

Ebenfalls strafrechtlich nicht uninteressant ist die Konstellation, in der der betroffene Arzt sich den Deckmantel einer Handelsgesellschaft gibt, welche nur die Aufgabe hat, den Bezug von Medizinprodukten teurer zu machen. Die Handelsgesellschaft bezieht die Medizinprodukte zum handelsüblichen Preis, gibt diese dann überteuert an den Arzt ab, der wiederum die überteuerte Rechnung gegenüber dem Kostenträger einreicht.

\section{Arten des Abrechnungsbetrugs}

Generell kann man die Abrechnungsbetrügereien unter den von mir als „goldene Drei" bezeichneten ersten in der Literatur aufgetretenen Deliktbeschreibungen zusammenfassen:

\section{Luftabrechnung}

Hier erfolgt die Zusetzung einer Gebührenordnungsposition, welche nicht im Ansatz erbracht wurde. Somit wird der Abrechnungsschein vor seiner Abgabe „aufgebläht“.

\section{Leistungssplitting}

In allen Gebührenordnungen gibt es Leistungsausschlüsse, also Leistungen, die nicht nebeneinander abgerechnet werden dürfen. Wenn es auch medizinisch möglich wäre, diese neben- bzw. nacheinander zu erbringen, darf doch nur die höher bewertete Leistung abgerechnet werden. Derartige Abrechnungsausschlüs- se werden von den die Abrechnungen kontrollierenden Regelwerken erkannt, und es erfolgt die Absetzung einer Ziffer. Leistungssplitting bezeichnet nunmehr die Tathandlung, durch welche die beiden Ziffern auf unterschiedliche Abrechnungstage gelegt werden, um das Regelwerk zu überlisten. Dadurch werden beide Leistungen vergütet, obwohl nur eine hätte vergütet werden dürfen.

Einen anderen Spezialfall des Leistungssplittings trifft man bei der Auflösung von Komplexleistungen an. Diese werden in ihre ebenfalls abrechnungsfähigen Einzelbestandteile zerlegt, welche abgerechnet zu einem höheren Honorar führen als die ursprüngliche Komplexleistung.

\section{Leistungsaufwertung}

Hier wird eine Leistung erbracht (z. B. kleiner Schienenverband mit Einschluss eines großen Gelenks), jedoch eine andere Leistung abgerechnet, z. B. ein großer Schienenverband unter Einschluss von 2 großen Gelenken.

Ein weiteres Beispiel könnte die Abrechnung von allgemeinen Heilbehandlungen zu besonderen Heilbehandlungen im Bereich der Unfallbehandlung sein. Dies könnte dann zum Tragen kommen, wenn der Patient sich direkt beim D-Arzt vorstellt und dieser vor der Entscheidung steht, die Art der Heilmethode zu wählen. (Die Überweisung eines Hausarztes indiziert ja bereits die besondere Heilbehandlung).

\section{Weitere Möglichkeiten}

So facettenreich das wirtschaftliche Handeln von Leistungserbringern in medizinischen Berufen ist, so vielschichtig sind auch die Möglichkeiten, das System in betrügerischer Absicht auszuhebeln. Von den vielen tatsächlich bei unseren Ermittlungen auffälligen Vorgehensweisen wie auch den theoretisch denkbaren Möglichkeiten soll hier im Zusammenhang mit der Abrechnung gegenüber Berufsgenossenschaften (BG) noch auf die Doppelabrechnung hingewiesen werden.

Es war in einem Ermittlungsfall festzustellen, dass alle BG-Fälle auch gegenüber der KV als ambulante Fälle abgerechnet wurden. Auf diese Weise wurden die seinerzeit geltenden Budgetbestimmungen ausgehebelt. Diese Form der Budgetausweitung fiel bei den Ermittlungsbehörden damals auch dadurch auf, dass bei allen BG-Patienten die gleiche Diagnose abgerechnet wurde (Deformität der Füße), sodass hierdurch das Schadensvolumen, in diesem Fall zu Lasten der KV, genau festgestellt werden konnte.

Natürlich erleichtert der Übertrag von Chipkartendaten die BG-Abrechnung ungemein, jedoch werden die Weichen für ein strafbares Handeln in dem Moment gestellt, in dem die automatische Systemabfrage erfolgt, ob ein Fall angelegt werden soll. Diese Frage grundsätzlich zu bejahen, birgt im geschilderten Zusammenhang große Gefahren.

\section{Ärztliche Dokumentation}

Ihr kommt im Zug von Ermittlungsverfahren große Bedeutung zu. Sie ist zunächst eine Berufspflicht. Sollte es sich um eine Leistungspflicht handeln, ist sie auch zwingend erforderlich, um die Leistung abrechnen zu können. Sofern die Leistungsbeschreibung expressis verbis die Dokumentation aufführt (z. B. „... einschließlich dokumentierter Nachweis des..."), wird sie auch von den Verfolgungsbehörden zwingend erwartet. $\mathrm{Ob}$ eine Dokumentation fachgerecht ist, wird nur dann eine Rolle spielen, wenn überhaupt etwas dokumentiert wurde. Fehlt die Dokumentation in den verpflichtenden Fällen, ist die Leistung nicht erbracht, auch wenn gegenüber dem Patienten ansonsten alles erfolgte, was die Leistungsbeschreibung anfordert.

Je umfangreicher dokumentiert wird, desto einfacher lassen sich Leistungserbringungen nachweisen, sodass man nicht oft genug auf eine peinlich ausführliche Dokumentation hinweisen kann.

\section{Korrespondenzadresse \\ J. Engelhard}

LKA 328 - Medicus, Landeskriminalamt Berlin

Columbiadamm 4, 10965 Berlin

lka@polizei.berlin.de

Interessenkonflikt. Der korrespondierende Autor gibt an, dass kein Interessenkonflikt besteht. 